\title{
Surveillance of Hepatocellular Carcinoma and Diagnostic Algorithms in Patients with Liver Cirrhosis
}

\author{
Jens U. Marquardta, b, c Marc Nguyen-Tata, b Peter R. Galle ${ }^{a, b}$ Marcus A. Wörnsa, b \\ ${ }^{a}$ I. Department of Internal Medicine, University Medical Center, Johannes Gutenberg University Mainz, Mainz, Germany; \\ ${ }^{b}$ Center for Cirrhosis Mainz (CCM), University Medical Center, Johannes Gutenberg University Mainz, Mainz, Germany; \\ ${ }^{\mathrm{c} B i o i n f o r m a t i c s}$ Core Facility Mainz (Bium-Mz), University Medical Center, Johannes Gutenberg University Mainz, Mainz, Germany
}

\section{Keywords}

Hepatocellular carcinoma · Screening · Diagnosis · Imaging

\section{Summary}

Background: Hepatocellular carcinoma (HCC) is the most deadly complication of all major chronic liver diseases. Since early detection is the most significant determinant of overall survival, intense screening is of major importance. Methods: This overview is based on a systematic review of the available literature on HCC screening and surveillance in the PubMed database. Results: Over the last decades, major etiological risk factors were identified and the population at highest risk for the development of HCC was clearly defined. Screening in these patients has been repeatedly demonstrated to detect early tumor stages and to be cost-effective. Therefore, screening is recommended by all current guidelines and usually comprises a bi-annual ultrasound examination in Western countries. In some Asian countries biomarkers are also used; however, their efficiency for Western HCCs remains to be determined. The detection of lesions $>1 \mathrm{~cm}$ during routine screening requires subsequent confirmation of HCC. The diagnosis can be accurately established by modern imaging techniques, i.e. computed tomography or magnetic resonance imaging, in the majority of patients. In ambiguous cases and if radiological criteria are not met by two imaging techniques, biopsies remain the gold standard for diagnosis. Furthermore, histology is of key importance for the development of new diagnostic and predictive biomarkers. Conclusion: Screening and detection algorithms for patients at risk for HCC are effective and should be rigorously implemented in clinical routine.

(C) 2016 S. Karger GmbH, Freiburg

\section{Introduction}

Hepatocellular carcinoma (HCC) is the fifth most common cancer in men and the seventh most common cancer in women worldwide, accounting for at least 600,000 deaths annually [1]. Incidence rates traditionally showed highest numbers in Southeast Asia and sub-Saharan Africa, mainly associated with chronic hepatitis B virus (HBV) infection. Generalized implementation of vaccination programs have proven to be effective in preventing infections in these countries and a constant decrease in newly diagnosed HCC has been observed during the last years. However, both incidence and mortality rates of HCC have doubled in the United States and in Europe during the past 2-3 decades and are predicted to rise continuously [2]. Although several confounding factors (e.g. immigration from high-incidence countries) contribute to increasing numbers in the Western world, together with pancreatic cancer and melanoma, HCC is currently among the fastest growing causes of cancer-related deaths in the USA.

The major etiologic agents responsible for chronic liver disease and subsequent development of cirrhosis (the major risk factor per se) as well as HCC are known and well characterized [2]. In Western countries, etiological agents comprise a heterogeneous group of chronic liver diseases that range from chronic viral hepatitis (e.g. infections with $\mathrm{HBV}$ and hepatitis $\mathrm{C}$ virus (HCV)) over ethanol abuse to non-alcoholic fatty liver disease/steatohepatitis (NAFLD/ $\mathrm{NASH}$ ) and other metabolic disorders. In particular, NAFLD/ NASH has become a relevant risk factor in Western countries due to an incline in prevalence and high numbers of HCCs without underlying cirrhosis $[3,4]$.

Due to our limited understanding of the tumor biology and the lack of curative treatment options, the most important prognostic factor still remains detection at early stages. Therefore, rigorous screening strategies for patients at risk are of particular importance. However, despite our increasing awareness of etiological risk factors, most patients present with advanced disease

\section{KARGER}

Fax +497614520714
Jun. Prof. Dr. med. Jens U. Marquardt

I. Department of Internal Medicine, University Medical Center

Johannes Gutenberg University Mainz

Langenbeckstraße 1, 55131 Mainz, Germany

marquarj@ uni-mainz.de 
Table 1. Patients at risk for HCC development (modified from $[5,8])$

\begin{tabular}{lll}
\hline Population at risk & $\begin{array}{l}\text { Incidence for efficacy of Incidence of HCC } \\
\text { surveillance }(>0.25 \text { life } \\
\text { year gained), \%/years }\end{array}$ \\
\hline Screening recommended & 0.2 & $0.4-0.6 \% /$ years \\
HBV-positive Asian male $>40$ years & 0.2 & $0.3-0.6 \% /$ years \\
HBV-positive Asian female $>50$ years & 0.2 & higher than without cirrhosis \\
HBV-positive with family history of HCC & 0.2 & younger age \\
HBV-positive African/North American blacks & $0.2-1.5$ & $3-8 \% /$ years \\
HBV-positive cirrhotic hepatitis & 1.5 & $3-5 \% /$ years \\
HCV-positive cirrhosis & 1.5 & $3-5 \% /$ years \\
Stage 4 primary biliary cirrhosis & 1.5 & unknown, but probably $>1.5 \% /$ years \\
Genetic hemochromatosis and cirrhosis & 1.5 & unknown, but probably $>1.5 \% /$ years \\
Alpha 1-antitrypsin deficiency and cirrhosis & 1.5 & unknown \\
Other cirrhosis & & $<0.2 \% /$ years \\
\hline Benefit uncertain & 0.2 & $<1.5 \% /$ years \\
HBV-positive $<40$ (males) or $<50$ (females) years & 1.5 & $<1.5 \% /$ years \\
HCV-positive and stage 3 fibrosis & 1.5 & \\
Non-cirrhotic NAFLD & &
\end{tabular}

stages at the time of diagnosis and less than $20 \%$ of the patients are eligible for curative treatment options, i.e. resection and/or liver transplantation [5]. These observations clearly highlight that HCC is a major health care problem in the Western world and underline the critical need for improved understanding of the molecular pathogenesis, development of diagnostic and predictive biomarkers, and identification of novel treatment options for this deadly disease [6]. The recommendations regarding screening and diagnosis of HCC are similar across international guidelines, such as those endorsed by the American Association for the Study of Liver Diseases (AASLD), the European Association of the Study of the Liver (EASL)/European Organization for Research and Treatment of Cancer (EORTC), or the Deutsche Gesellschaft für Gastroenterologie, Verdauungs- und Stoffwechselerkrankungen (DGVS). In this systematic review, we will primarily focus on these three guidelines which are widely implemented in Western countries.

\section{Identification of Patients at Risk: The Target Population for Screening}

The overall goal of successful screening is effectiveness, i.e., the method needs to result in a noticeable benefit for clinical practice [7]. Therefore, screening needs to be available to a broader population of patients and should effectively detect early stages where curative treatment is available. This also implies that the level of risk for each subgroup of patients needs to be defined to make screening worthwhile [5]. By current definitions, screening is considered effective if the yielded increase in life time of the screening population is at least 3 months [8]. In this context, identification of the patients at highest risk for development of HCC is a major objective, and screening is currently recommended for patients whose annual risk of HCC development exceeds $1.5-2 \%$ (table 1) [5, 9].
Since bridging fibrosis and cirrhosis are well established predisposing factors for HCC development, cirrhotic patients are the ideal population for screening. Accordingly, guidelines of the American, European, and German liver/gastroenterology associations (i.e. AASLD, EASL, DGVS) universally recommend screening in all patients with liver cirrhosis, regardless of the etiology $[5,10,11]$. Notably, the Asian Pacific Association for the Study of the Liver (APASL) is more restrictive and limits the screening to patients with cirrhosis due to viral hepatitis [12].

High incidence rates of HCC are also observed in patients with chronic HBV infection, even in the absence of cirrhosis. Consequently, screening of this subgroup of patients depends on the incidence of HBV infections in the respective regions. The AASLD guidelines recommend screening for Asian males older than 40 years and for women older than 50 years [5]. Additionally, screening should be performed for patients with a family history of HCC and for African American blacks older than 20 years. The EASL guidelines are more restrictive and identify HBV patients with chronic active HBV or a family history of HCC as the only candidates for screening [10].

Given the high incidence of HCC in non-cirrhotic NASH, current German guidelines additionally recommend screening for NASH patients $[4,11]$. However, data ultimately supporting this approach as well as rigorous cost-effectiveness analyses in this large, heterogeneous patient population are still lacking [13]. Some recent evidence suggests that risk stratification of NASH patients based on genetic susceptibility (e.g. by PNPLA3) might increase the effectiveness of the screening, but this observation also needs confirmation [14]. Notably, to better predict the risk of HCC development in different patient populations, several score systems have been developed [15-17]. The best validated score for chronic HBV-infected patients is the REACH-B score $[16,18]$. Despite its potential of identifying most suitable candidates for screening, external validation in large cohorts is still needed. 
An unresolved question is to what extent currently recommended strategies negatively impair cost-effectiveness when diagnosis of cirrhosis or bridging fibrosis is misclassified due to noninvasive testing. This method is frequently applied to patients with $\mathrm{HCV}$, a population at high risk for HCC development [19]. Given the high success rate of novel HCV therapies leading to at least a fourfold reduction of HCC, however, incidences in cirrhotic patients, screening of these patients, and guideline recommendations will likely be modified during the next few years [20].

\section{Effectiveness, Method, and Interval of Screening}

As already stated, screening should be generally recommended when the HCC incidence exceeds $1.5-2 \%$. The effectiveness of HCC surveillance in clinical routine largely depends on adherence to screening and sensitivity of surveillance methods for early detection in patients at risk. Generally, screening utilization and sensitivity must exceed 34 and $42 \%$, respectively, to provide a benefit for patient outcome [21]. Several analyses have confirmed the cost-effectiveness of screening for HCC in different patient populations $[22,23$.

Overall, consent among all these studies was that screening for HCC is cost-effective when using abdominal ultrasound. Since ultrasound is widely available, accurate, non-invasive, and inexpensive, it is the backbone of all current guidelines [5, 10-12]. Sensitivity of the method is highly dependent on the investigator and ranges from 29 to $100 \%$ [24, 25]. While a meta-analysis recently demonstrated a pooled sensitivity of only $63 \%$ (95\% confidence interval: $49-76 \%)$ in cirrhotic patients, sensitivity can be significantly increased when performed by trained experts $[10,25]$. Therefore, it is generally accepted that screening should be performed by experienced centers [10, 25, 26].

Several studies addressed the optimal screening interval in cirrhotic patients whereby an interval of 6 months significantly improved the sensitivity of early HCC detection compared to 12 months (70 vs. 50\%) [27-29]. Importantly, considering both current data demonstrating that a reduction of the screening interval from 6 to 3 months did not yield an increased detection of early lesions or patients eligible for curative treatment as well as the predicted doubling time of the tumor, there is no rationale for shortening this interval [30]. Altogether, a 6-monthly screening interval can be considered most appropriate and cost-effective in patients at high risk for HCC development [31].

In cirrhotic livers with heterogeneous and nodular parenchyma, detection of HCC can be challenging when using ultrasound [32]. Screening is further compromised by obesity of the patients, in particular of NAFLD/NASH patients. Therefore, computed tomography (CT) as well as magnetic resonance imaging (MRI) have been proposed as alternative screening modalities to overcome these obstacles and to improve the detection rate for small HCCs. However, gain in accuracy needs to outperform both the additional costs as well as the potential risk for the patients (e.g. radiation exposure). While the role of these cross-sectional imaging techniques for diagnosis of HCC have been well established, their effectiveness for screening is less well studied [5]. Head-to-head studies showed that bi-annual ultrasound has a similar detection rate of early HCC as well as lower costs compared to annual CT (ultrasound: USD 17,041.- vs. CT: USD 57,383.-) [33]. In conclusion, bi-annual ultrasound is the most cost-effective screening approach for HCC with a similar sensitivity compared to cross-sectional imaging techniques but no relevant risks. Other imaging techniques should only be applied if ultrasound assessment is compromised.

\section{Biomarkers for Screening and Detection}

Serological tests for early HCC detection in patients at risk have been intensively studied, and some showed promising possibilities of improving the detection rates of small HCCs [34]. Among those, the best studied biomarker for HCC is alpha-fetoprotein (AFP) [35]. Although several reports demonstrated that AFP, in combination with ultrasound, can increase early detection, elevation of serum AFP can occur in cirrhotic patients even in the absence of malignancy. High AFP levels are also observed in cirrhosis with high inflammatory activity and may correlate with aminotransferase levels [36, 37]. A cut-off value of $20 \mathrm{ng} / \mathrm{ml}$ was shown to optimally level sensitivity and specificity of AFP; however, sensitivity remained at only $60 \%[38,39]$. Of note, reducing the cut-off resulted in a considerable number of false-positive findings which would require additional and, frequently, unnecessary tests. Therefore, the AASLD and EASL guidelines consider specificity and sensitivity of AFP to be insufficient as a good screening test and, therefore, do not endorse its use in clinical routine, neither alone nor in combination with ultrasound $[5,10]$.

Other biomarkers evaluated in clinical trials for HCC are lectinreactive AFP (AFP-L3), des-gamma-carboxy prothrombin (DCP), golgi protein-73 (GP73), and osteopontin (OPN) [34, 40]. While results for some of these markers are promising, prospective studies as well as independent validation in large cohorts of patients with different risk factors are required before their value for HCC screening can be accurately estimated [7].

\section{Prevention of Liver-Related Mortality}

As described in the above sections, screening is uniformly recommended in all national and international guidelines for HCC. A crucial issue is to what extent the recommended screening strategies are effectively reducing HCC-related mortality. Although the current evidence does not provide a definitive answer to this question and available studies are frequently not of the highest quality, most studies reach the consensus that screening is beneficial $[9$, 41]. Considering that patients who develop symptoms from HCC generally present with advanced stages when treatment options are extremely limited, screening seems indispensable and should be rigorously implemented. 
Fig. 1. Diagnostic algorithm and recall policy (modified from [10]). ${ }^{*}$ One imaging technique only recommended in centers of excellence with high-end radiological equipment; ${ }^{* *}$ HCC radiological features: arterial hypervascularization (washin) and portal-venous/delayed phase wash-out. US = Ultrasound; $\mathrm{CT}$ = computed tomography; $\mathrm{MRI}$ = magnetic resonance imaging; HCC = hepatocellular carcinoma.

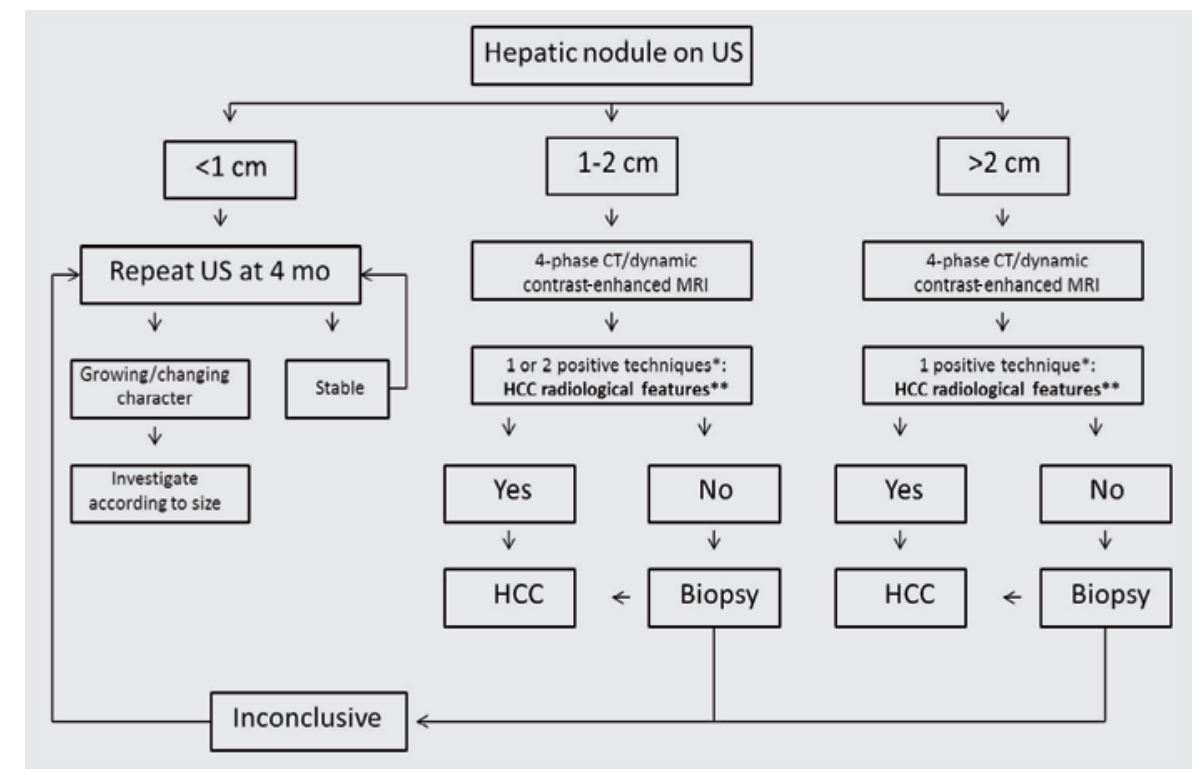

\section{Diagnostic Approach to Hepatocellular Carcinoma}

\section{Recall Policy}

A stringent recall policy algorithm is crucial when an abnormal finding (i.e. a newly focal hepatic nodule/mass, known focal hepatic nodule with changing echo patterns or growth) is detected during routine ultrasound screening with the aim to diagnose HCC at an early stage when curative treatment approaches can be applied [10, 42]. Pathology studies have shown that the majority of nodules smaller than $1 \mathrm{~cm}$ detected in a cirrhotic liver are not HCCs [43], and according to the current EASL and AASLD guidelines, nodules less than $1 \mathrm{~cm}$ in diameter should therefore be followed up every 3 (AASLD) to 4 (EASL) months by conventional ultrasound for the first year and every 6 months thereafter (fig. 1) $[5,10,43]$. However, the detection of nodules larger than $1 \mathrm{~cm}$ during routine ultrasound screening requires further investigation and subsequent confirmation of HCC either by means of non-invasive criteria or by biopsy, as described in the following sections (fig. 1) $[5,10]$. The latter approach is recommended by the DGVS guideline independent of the size of the nodule (i.e. also in suspicious nodules smaller than $1 \mathrm{~cm}$ ) [11].

\section{Non-Invasive Diagnosis Criteria}

Due to limitations of ultrasound- or CT-guided biopsy and pathological diagnosis (feasibility, risk of bleeding, or needle track seeding [44]; differentiation between high-grade dysplastic nodules and early HCCs [43]), the guidelines on the clinical management of HCC endorsed by the EASL in 2001 have already reported noninvasive criteria for the diagnosis of HCC in a cirrhotic liver based on a combination of imaging and laboratory findings [10, 45]. Coincidental findings of contrast uptake in the arterial phase (hypervascularization) of two dynamic contrast-enhanced imaging techniques (CT, MRI, angiography, or ultrasound) in nodules larger than $2 \mathrm{~cm}$ were considered diagnostic, or alternatively, one imaging technique together with an elevated level of AFP (above 400 $\mathrm{ng} / \mathrm{ml}$ ). If atypical features were seen on one imaging technique, then an alternative dynamic imaging technique should be performed. Only if these conditions were not fulfilled, a biopsy was mandatory [45]. In 2005, the AASLD guidelines established the currently valid radiological features of HCC, i.e. wash-in in the arterial phase and wash-out in the portal-venous or delayed phase of a dynamic contrast-enhanced imaging technique (CT, MRI, or US) [46-48]. These recommendations were based again on the fact that HCC receives its vascular supply predominantly via the hepatic artery (hypervascularization in the arterial phase). As a further consequence, wash-out in the portal-venous or delayed phase is observed in comparison with the surrounding liver tissue. HCC diagnosis was established in nodules larger than $2 \mathrm{~cm}$ showing these features in one dynamic contrast-enhanced imaging technique or in nodules of $1-2 \mathrm{~cm}$ in diameter in two alternative imaging techniques. The AFP elevation was dropped from the diagnostic agenda because AFP determination lacks adequate sensitivity and specificity for effective surveillance and diagnosis $[10,46]$. The latest update of the AASLD guidelines has even proposed that one imaging technique (CT or MRI) showing radiological hallmarks of HCC is sufficient for the non-invasive diagnosis of nodules of 1-2 $\mathrm{cm}$ in diameter [5]. At that time, contrast-enhanced ultrasound was not considered for the diagnostic agenda due to false-positive results in patients with cholangiocarcinoma $[5,49]$. The current update of the EASL guidelines from 2012 has finally confirmed this approach; however, it includes the restriction that the one imaging technique rule in nodules of $1-2 \mathrm{~cm}$ in diameter can only be applied in optimal settings (4-phase multidetector CT and/or dynamic contrast-enhanced MRI following reported protocols and local skills at the high-end level) [10].

Only in case of uncertainty or inconclusive/atypical radiological findings in both dynamic contrast-enhanced imaging techniques (CT and MRI), diagnosis should be confirmed by biopsy (fig. 1) [5, 10]. Again, in contrast to the current EASL and AASLD guidelines, this approach is recommended by the DGVS guideline independ- 
ent of the size of the nodule (i.e. also in suspicious nodules smaller than $1 \mathrm{~cm}$ ). In addition, contrast-enhanced ultrasound can furthermore be used as an alternative dynamic imaging technique [11].

\section{Pathological Diagnosis}

Pathological diagnosis of HCC is recommended for suspicious nodules occurring in patients with non-cirrhotic liver detected during routine screening ultrasound, and for cirrhotic patients with inconclusive or atypical findings in two (fig. 1) or three contrast-enhanced imaging techniques $[5,10,11]$. However, biopsy of small, atypical lesions is associated with a high rate of false-negative findings due to sampling error [10]. A second biopsy is potentially recommended in case of inconclusive findings as well as growth or change in enhancement pattern identified in the setting of a tight follow-up every 3 months $[5,10,11]$. In this context, the incidence of needle tract tumor seeding following biopsy of a HCC was reported to be $2.7 \%$ overall or $0.9 \%$ per year [44].

Interpretation of biopsies and distinction between high-grade dysplastic nodules and early HCC is challenging [43]. Biopsies should therefore be assessed by an expert hepato-pathologist [5, 10]. If tissue is not clearly HCC, immunostaining for glypican 3 (GPC3), heat shock protein 70 (HSP70), and glutamine synthetase (GS) is recommended by all guidelines to improve accuracy $[5,10$, $11,50]$. Additional staining can be considered to detect progenitor cell features (K19 and EpCAM) or to assess neovascularization (CD34) $[5,10,11,43]$. It is worth mentioning that, apart from diagnosis, histology is of key importance for the development of new diagnostic and predictive biomarkers.

\section{Conclusion and Outlook}

During the last 15 years, our understanding of the pathophysiology of chronic liver diseases and subsequent hepatocarcinogenesis has dramatically increased. Several studies led to the identifica- tion of patients at high risk for the development of HCC. As a result, all current guidelines implemented rigorous screening and detection algorithms for these patients. Progress in imaging techniques further enabled clinicians to accurately diagnose HCC at early and potentially curative stages of the disease course and, thus, provided a measurable improvement in the medical treatment of patients at risk. However, adherence to these screening programs is still suboptimal and detailed information on the potentially reduced HCC-related mortality and the effectiveness of these measures are lacking. Additionally, despite effective screening programs, a considerable number of patients are still diagnosed at advanced stages, and prospective validation of promising non-invasive candidate biomarkers for the early detection of HCC in large cohorts to complement the screening programs is still lacking. Therefore, our efforts should still focus on the translation of knowledge (bench-to-bedside as well as bedside-to-bench) into clinical practice.

Provided that the life-time risk for HCC development is considerably high and, for most HCCs, highly influenced by environmental factors (e.g. HCV infections), prevention and early treatment of the underlying liver disease should remain the major goal [51].

\section{Acknowledgement}

This work was supported by internal funding from the University of Mainz, Medical Center. J.U. Marquardt is supported by grants from the German Cancer Aid (DKH 110989) and the Volkswagen Foundation (Lichtenberg program).

\section{Disclosure Statement}

M.A. Wörns and P.R. Galle have received consulting and lecture fees from Bayer HealthCare, Lilly, and Bristol-Myers Squibb. M. Nguyen-Tat has received consulting and lecture fees from Bayer HealthCare and Bristol-Myers Squibb. J.U. Marquardt has nothing to disclose.

\section{References}

1 El-Serag HB: Epidemiology of viral hepatitis and hepatocellular carcinoma. Gastroenterology 2012;142:12641273.e1261.

2 El-Serag HB: Hepatocellular carcinoma. N Engl J Med 2011;365:1118-1127.

3 Marquardt JU, Galle PR, Teufel A: Molecular diagnosis and therapy of hepatocellular carcinoma (HCC): an emerging field for advanced technologies. J Hepatol 2012;56:267-275.

4 Ascha MS, Hanouneh IA, Lopez R, Tamimi TA, Feldstein $\mathrm{AF}$, Zein NN: The incidence and risk factors of hepatocellular carcinoma in patients with nonalcoholic steatohepatitis. Hepatology 2010;51:1972-1978.

5 Bruix J, Sherman M; American Association for the Study of Liver Diseases: Management of hepatocellular carcinoma: an update. Hepatology 2011;53:1020-1022.

6 Marquardt JU, Andersen JB, Thorgeirsson SS: Functional and genetic deconstruction of the cellular origin in liver cancer. Nat Rev Cancer 2015;15:653-667.
Singal AG, El-Serag HB: Hepatocellular carcinoma from epidemiology to prevention: translating knowledge into practice. Clin Gastroenterol Hepatol 2015; 13:2140-2151.

8 Simpson HN, McGuire BM: Screening and detection of hepatocellular carcinoma. Clin Liver Dis 2015;19: 295-307.

9 Sherman M, Colombo M: Hepatocellular carcinoma screening and diagnosis. Semin Liver Dis 2014;34:389397.

10 European Association For The Study Of The Liver, European Organisation For Research and Treatment Of Cancer: EASL-EORTC clinical practice guidelines: management of hepatocellular carcinoma. J Hepatol 2012;56:908-943.

11 Greten TF, Malek NP, Schmidt S, et al: Diagnosis of and therapy for hepatocellular carcinoma (Article in German). Z Gastroenterol 2013;51:1269-1326.
12 Omata M, Lesmana LA, Tateishi R, et al: Asian Pacific Association for the Study of the Liver consensus recommendations on hepatocellular carcinoma. Hepatol Int 2010;4:439-474.

13 Pocha C, Kolly P, Dufour JF: Nonalcoholic fatty liver disease-related hepatocellular carcinoma: a problem of growing magnitude. Semin Liver Dis 2015;35:304-317.

14 Singal AG, Manjunath H, Yopp AC, et al: The effect of PNPLA3 on fibrosis progression and development of hepatocellular carcinoma: a meta-analysis. Am J Gastroenterol 2014;109:325-334.

15 Lok AS, Seeff LB, Morgan TR, et al: Incidence of hepatocellular carcinoma and associated risk factors in hepatitis C-related advanced liver disease. Gastroenterology 2009;136:138-148.

16 Yang HI, Yuen MF, Chan HL, et al: Risk estimation for hepatocellular carcinoma in chronic hepatitis B (REACH-B): development and validation of a predictive score. Lancet Oncol 2011;12:568-574. 
17 Flemming JA, Yang JD, Vittinghoff E, Kim WR, Terrault NA: Risk prediction of hepatocellular carcinoma in patients with cirrhosis: the ADRESS-HCC risk model. Cancer 2014;120:3485-3493.

18 Chen TM, Chang CC, Huang PT, Wen CF, Lin CC: Performance of risk estimation for hepatocellular carcinoma in chronic hepatitis B (REACH-B) score in classifying treatment eligibility under 2012 Asian Pacific Association for the Study of the Liver (APASL) guideline for chronic hepatitis B patients. Aliment Pharmacol Ther 2013;37:243-251.

19 Castera L, Vergniol J, Foucher J, et al: Prospective comparison of transient elastography, Fibrotest, APRI, and liver biopsy for the assessment of fibrosis in chronic hepatitis C. Gastroenterology 2005;128:343-350.

20 Nahon P, Bourcier V, Layese R, et al: Incidence, characteristics and determinants of primary liver cancer occurring during surveillance of compensated HCV cirrhosis according to sustained virological response (ANRS CO12 CirVir). J Hepatol 2015;62:S432.

21 Mourad A, Deuffic-Burban S, Ganne-Carrie N, et al: Hepatocellular carcinoma screening in patients with compensated hepatitis $\mathrm{C}$ virus (HCV)-related cirrhosis aware of their HCV status improves survival: a modeling approach. Hepatology 2014;59:1471-1481.

22 Cucchetti A, Cescon M, Erroi V, Pinna AD: Cost-effectiveness of liver cancer screening. Best Pract Res Clin Gastroenterol 2013;27:961-972.

23 Bolondi L, Sofia S, Siringo S, et al: Surveillance programme of cirrhotic patients for early diagnosis and treatment of hepatocellular carcinoma: a cost effectiveness analysis. Gut 2001;48:251-259.

24 Seitz K: Quality of abdominal ultrasound. Ultraschal Med 2006;27:217-219.

25 Singal A, Volk ML, Waljee A, et al: Meta-analysis: surveillance with ultrasound for early-stage hepatocellular carcinoma in patients with cirrhosis. Aliment Pharmacol Ther 2009;30:37-47.

26 Braillon A: Is the American Association for the Study of Liver Diseases recommendation for hepatocellular carcinoma screening a cul-de-sac? World J Gastroenterol 2013;19:3369-3370.

27 Trevisani F, De Notariis S, Rapaccini G, et al: Semiannual and annual surveillance of cirrhotic patients for hepatocellular carcinoma: effects on cancer stage and patient survival (Italian experience). Am J Gastroenterol 2002;97:734-744.
28 Zhang BH, Yang BH, Tang ZY: Randomized controlled trial of screening for hepatocellular carcinoma. J Cancer Res Clin Oncol 2004;130:417-422.

29 Santi V, Trevisani F, Gramenzi A, et al: Semiannual surveillance is superior to annual surveillance for the detection of early hepatocellular carcinoma and patient survival. J Hepatol 2010;53:291-297.

30 Trinchet JC, Chaffaut C, Bourcier V, et al: Ultrasonographic surveillance of hepatocellular carcinoma in cirrhosis: a randomized trial comparing 3- and 6-month periodicities. Hepatology 2011;54:1987-1997.

31 Thompson Coon J, Rogers G, Hewson P, et al: Surveillance of cirrhosis for hepatocellular carcinoma: a costutility analysis. Br J Cancer 2008;98:1166-1175.

32 Del Poggio P, Olmi S, Ciccarese F, et al: Factors that affect efficacy of ultrasound surveillance for early stage hepatocellular carcinoma in patients with cirrhosis. Clin Gastroenterol Hepatol 2014;12:1927-1933.e1922.

33 Pocha C, Dieperink E, McMaken KA, Knott A, Thuras P, Ho SB: Surveillance for hepatocellular cancer with ultrasonography vs. computed tomography - a randomised study. Aliment Pharmacol Ther 2013;38:303312.

34 Bertino G, Ardiri A, Malaguarnera M, Malaguarnera G, Bertino N, Calvagno GS: Hepatocellular carcinoma serum markers. Semin Oncol 2012;39:410-433.

35 Sherman M: Alphafetoprotein: an obituary. J Hepatol 2001;34:603-605.

36 Singal AG, Conjeevaram HS, Volk ML, et al: Effectiveness of hepatocellular carcinoma surveillance in patients with cirrhosis. Cancer Epidemiol Biomarkers Prev 2012;21:793-799.

37 Di Bisceglie AM, Sterling RK, Chung RT, et al: Serum alpha-fetoprotein levels in patients with advanced hepatitis C: results from the HALT-C Trial. J Hepatol 2005;43:434-441.

38 El-Serag HB, Kanwal F, Davila JA, Kramer J, Richardson P: A new laboratory-based algorithm to predict development of hepatocellular carcinoma in patients with hepatitis C and cirrhosis. Gastroenterology 2014; 146:1249-1255.e1241.

39 Trevisani F, D'Intino PE, Morselli-Labate AM, et al: Serum alpha-fetoprotein for diagnosis of hepatocellular carcinoma in patients with chronic liver disease: influence of HBsAg and anti-HCV status. J Hepatol 2001;34:570-575.

40 Marrero JA, Feng Z, Wang Y, et al: Alpha-fetoprotein, des-gamma carboxyprothrombin, and lectin-bound alpha-fetoprotein in early hepatocellular carcinoma. Gastroenterology 2009;137:110-118.
41 Kansagara D, Papak J, Pasha AS, et al: Screening for hepatocellular carcinoma in chronic liver disease: a systematic review. Ann Intern Med 2014;161:261-269.

42 Lencioni R, Llovet JM: Modified RECIST (mRECIST) assessment for hepatocellular carcinoma. Semin Liver Dis 2010;30:52-60.

43 Roskams T: Anatomic pathology of hepatocellular carcinoma: impact on prognosis and response to therapy. Clin Liver Dis 2011;15:245-259, vii-X.

44 Silva MA, Hegab B, Hyde C, Guo B, Buckels JA, Mirza DF: Needle track seeding following biopsy of liver lesions in the diagnosis of hepatocellular cancer: a systematic review and meta-analysis. Gut 2008;57:15921596

45 Bruix J, Sherman M, Llovet JM, et al: Clinical management of hepatocellular carcinoma. Conclusions of the Barcelona-2000 EASL conference. European Association for the Study of the Liver. J Hepatol 2001;35:421430 .

46 Bruix J, Sherman M; Practice Guidelines Committee, American Association for the Study of Liver Diseases: Management of hepatocellular carcinoma. Hepatology 2005; 42:1208-1236.

47 Burrel M, Llovet JM, Ayuso C, et al: MRI angiography is superior to helical CT for detection of HCC prior to liver transplantation: an explant correlation. Hepatology 2003;38:1034-1042.

48 Yu JS, Kim KW, Kim EK, Lee JT, Yoo HS: Contrast enhancement of small hepatocellular carcinoma: usefulness of three successive early image acquisitions during multiphase dynamic MR imaging. AJR Am J Roentgenol 1999;173:597-604.

49 Vilana R, Forner A, Bianchi L, et al: Intrahepatic peripheral cholangiocarcinoma in cirrhosis patients may display a vascular pattern similar to hepatocellular carcinoma on contrast-enhanced ultrasound. Hepatology 2010;51:2020-2029.

50 International Consensus Group for Hepatocellular Neoplasia: Pathologic diagnosis of early hepatocellular carcinoma: a report of the international consensus group for hepatocellular neoplasia. Hepatology 2009; 49:658-664.

51 Tomasetti C, Vogelstein B: Cancer etiology. Variation in cancer risk among tissues can be explained by the number of stem cell divisions. Science 2015;347:78-81. 